Preprint SSU-HEP-00/05

Samara State University

\title{
PROTON POLARIZABILITY CONTRIBUTION TO THE HYDROGEN HYPERFINE SPLITTING
}

\author{
R.N. Faustov \\ Scientific Council "Cybernetics" RAS \\ 117333, Moscow, Vavilov, 40, Russia, \\ A.P. Martynenko \\ Department of Theoretical Physics, Samara State University, \\ 443011, Samara, Pavlov, 1, Russia
}

\begin{abstract}
The contribution of the proton polarizability to the hydrogen hyperfine splitting is evaluated on the basis of modern experimental and theoretical results on the proton polarized structure functions. The value of this correction is equal to $1.4 \mathrm{ppm}$.
\end{abstract}


The investigation of the hyperfine splitting (HFS) of the hydrogen atom ground state is considered during many years as an important test of quantum electrodynamics. The experimental value of the hydrogen hyperfine splitting was obtained with very high accuracy [1]:

$$
\Delta E_{H F S}^{\exp }=1420405.7517667(9) \mathrm{kHz} .
$$

The corresponding theoretical expression of the hydrogen hyperfine splitting may be written at present time in the form [2]:

$$
\Delta E_{H F S}^{t h}=E_{F}\left(1+\delta^{Q E D}+\delta^{S}+\delta^{P}\right), \quad E_{F}=\frac{8}{3} \alpha^{4} \frac{\mu_{P} m_{p}^{2} m_{e}^{2}}{\left(m_{p}+m_{e}\right)^{3}},
$$

where $\mu_{P}$ is the proton magnetic moment, $m_{e}, m_{p}$ are the masses of the electron and proton. The calculation of different corrections to $E_{F}$ has a long history. Modern status in the theory of hydrogenic atoms was presented in details in [3]. $\delta^{Q E D}$ denotes the contribution of higher-order quantumelectrodynamical effects. Corrections $\delta^{S}$ and $\delta^{P}$ take into account the influence of strong interaction. $\delta^{S}$ describes the effects of proton finite-size and recoil contribution. $\delta^{P}$ is the correction due to the proton polarizability. Basic uncertainty of theoretical result (2) is related with this term.

The main contribution to $\delta^{P}$ is determined by two-photon diagrams, shown in Figure 1. The corresponding amplitudes of virtual Compton scattering on proton can be expressed through nucleon polarized structure functions $\mathrm{G}_{1}\left(\nu, \mathrm{Q}^{2}\right)$ and $\mathrm{G}_{2}\left(\nu, \mathrm{Q}^{2}\right)$. Inelastic contribution of the diagrams (a), (b) Figure 1 may be presented in the form [3-8]:

$$
\begin{gathered}
\Delta E_{H F S}^{P}=\frac{Z \alpha m_{e}}{2 \pi m_{p}(1+\kappa)} E_{F}\left(\Delta_{1}+\Delta_{2}\right)=\left(\delta_{1}^{P}+\delta_{2}^{P}\right) E_{F}=\delta^{P} E_{F}, \\
\Delta_{1}=\int_{0}^{\infty} \frac{d Q^{2}}{Q^{2}}\left\{\frac{9}{4} F_{2}^{2}\left(Q^{2}\right)-4 m_{p}^{3} \int_{\nu_{t h}}^{\infty} \frac{d \nu}{\nu} \beta_{1}\left(\frac{\nu^{2}}{Q^{2}}\right) G_{1}\left(\nu, Q^{2}\right)\right\}, \\
\Delta_{2}=-12 m_{p}^{2} \int_{0}^{\infty} \frac{d Q^{2}}{Q^{2}} \int_{\nu_{t h}}^{\infty} d \nu \beta_{2}\left(\frac{\nu^{2}}{Q^{2}}\right) G_{2}\left(\nu, Q^{2}\right),
\end{gathered}
$$

where $\nu_{t h}$ determines the pion-nucleon threshold:

$$
\nu_{t h}=m_{\pi}+\frac{m_{\pi}^{2}+Q^{2}}{2 m_{p}}
$$

and the functions $\beta_{1,2}$ have the form:

$$
\begin{gathered}
\beta_{1}(\theta)=3 \theta-2 \theta^{2}-2(2-\theta) \sqrt{\theta(\theta+1)}, \\
\beta_{2}(\theta)=1+2 \theta-2 \sqrt{\theta(\theta+1)}, \quad \theta=\nu^{2} / Q^{2} .
\end{gathered}
$$

$F_{2}\left(Q^{2}\right)$ is the Pauli form factor of the proton, $\kappa$ is the proton anomalous magnetic moment: $\kappa=1.792847386(63)$ [9]. During many years there was not enough 

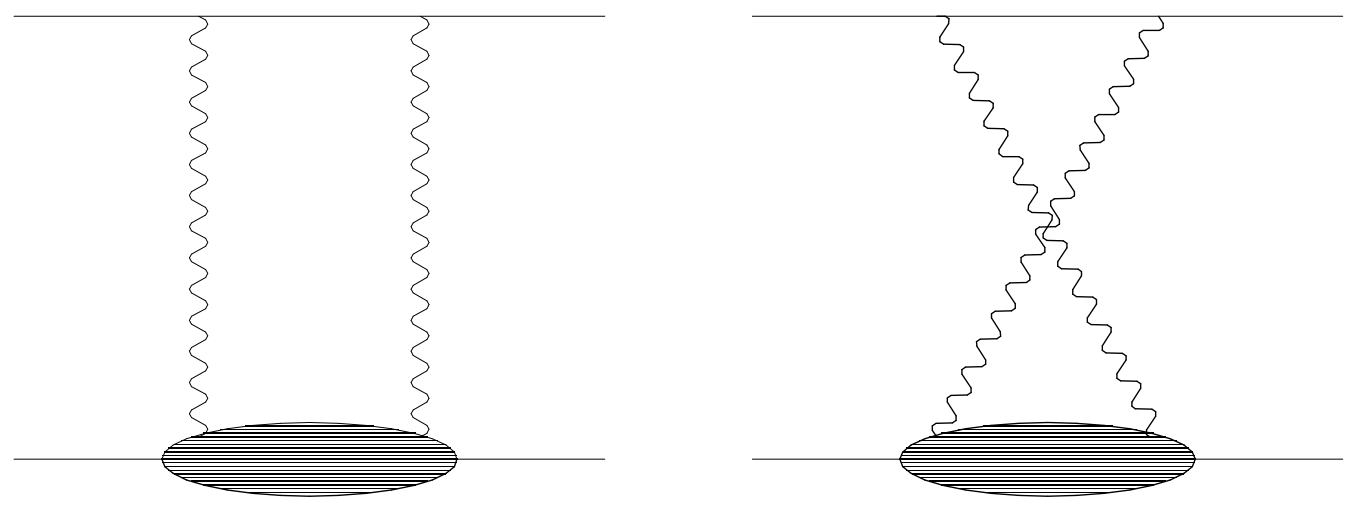

Figure 1: Feynman diagrams for proton polarizability correction to the hydrogen HFS

experimental data and theoretical information about proton spin-dependent structure functions. So, the previous study of the contribution $\Delta E_{H F S}^{P}$ contains only estimation of the proton polarizability effects: $\delta^{P} \sim 1 \div 2$ ppm or the calculation of main resonance contributions [6, 7, 8, 10]. The theoretical bound for the proton polarizability contribution is $\left|\delta^{P}\right| \leq 4 \mathrm{ppm}$. As noted in [3], the problem of the proton polarizability contribution requires new investigation, which takes into account more recent experimental data on the spin structure of the nucleon.

The polarized structure functions $g_{1}\left(\nu, Q^{2}\right)$ and $g_{2}\left(\nu, Q^{2}\right)$ enter in the antisymmetric part of hadronic tensor $W_{\mu \nu}$, describing lepton-nucleon deep inelastic scattering [11]:

$$
\begin{gathered}
W_{\mu \nu}=W_{\mu \nu}^{[S]}+W_{\mu \nu}^{[A]} \\
W_{\mu \nu}^{[S]}=\left(-g_{\mu \nu}+\frac{q_{\mu} q_{\nu}}{q^{2}}\right) W_{1}\left(\nu, Q^{2}\right)+\left(P_{\mu}-\frac{P \cdot q}{q^{2}} q_{\mu}\right)\left(P_{\nu}-\frac{P \cdot q}{q^{2}} q_{\nu}\right) \frac{W_{2}\left(\nu, Q^{2}\right)}{m_{p}^{2}}, \\
W_{\mu \nu}^{[A]}=\epsilon_{\mu \nu \alpha \beta} q^{\alpha}\left\{S^{\beta} \frac{g_{1}\left(\nu, Q^{2}\right)}{P \cdot q}+\left[(P \cdot q) S^{\beta}-(S \cdot q) P^{\beta}\right] \frac{g_{2}\left(\nu, Q^{2}\right)}{(P \cdot q)^{2}}\right\}
\end{gathered}
$$

where $g_{1}\left(\nu, Q^{2}\right)=m_{p}^{2} \nu G_{1}\left(\nu, Q^{2}\right), g_{2}\left(\nu, Q^{2}\right)=m_{p} \nu^{2} G_{2}\left(\nu, Q^{2}\right), q^{2}=-Q^{2}$ is the square of the four-momentum transfer. The invariant quantity $P \cdot q$ is related to the energy transfer $\nu$ in the proton rest frame: $P \cdot q=m_{p} \nu$. The invariant mass of the electroproduced hadronic system, $\mathrm{W}$, is then $W^{2}=m_{p}^{2}+2 m_{p} \nu-Q^{2}$.

The proton spin structure functions can be measured in the inelastic scattering of polarized electrons on polarized protons. Recent improvements in polarized lepton beams and targets have made it possible to make increasingly accurate measurements of nucleon polarized structure functions $g_{1,2}$ in experiments at SLAC, CERN and DESY [12-19]. The spin dependent structure functions may be expressed in terms of virtual photon-absorption cross-sections [11]:

$$
g_{1}\left(\nu, Q^{2}\right)=\frac{m_{p} \cdot K}{8 \pi^{2} \alpha\left(1+Q^{2} / \nu^{2}\right)}\left[\sigma_{1 / 2}\left(\nu, Q^{2}\right)-\sigma_{3 / 2}\left(\nu, Q^{2}\right)+\frac{2 \sqrt{Q^{2}}}{\nu} \sigma_{T L}\left(\nu, Q^{2}\right)\right]
$$




$$
g_{2}\left(\nu, Q^{2}\right)=\frac{m_{p} \cdot K}{8 \pi^{2} \alpha\left(1+Q^{2} / \nu^{2}\right)}\left[-\sigma_{1 / 2}\left(\nu, Q^{2}\right)+\sigma_{3 / 2}\left(\nu, Q^{2}\right)+\frac{2 \nu}{\sqrt{Q^{2}}} \sigma_{T L}\left(\nu, Q^{2}\right)\right]
$$

where $K=\nu-\frac{Q^{2}}{2 m_{p}}$ is Hand kinematical flux factor for virtual photons, $\sigma_{1 / 2}, \sigma_{3 / 2}$ are the virtual photoabsorption transverse cross sections for total helicity between photon and nucleon of $1 / 2$ and $3 / 2$ respectively, $\sigma_{T L}$ is the interference term between the transverse and longitudinal photon-nucleon amplitudes. In this work we calculate contribution $\Delta E_{H F S}^{P}$ on the basis of modern experimental data on structure functions $g_{1,2}\left(\nu, Q^{2}\right)$ and theoretical predictions on cross sections $\sigma_{1 / 2,3 / 2, T L}$.

To obtain correction (3) at the resonance region $\left(W^{2} \leq 4 G e V^{2}\right)$ we use the Breit-Wigner parameterization for the photoabsorption cross sections in (12), (13), suggested in [20, 21, 22, 23, 24, 25, 26]. There are many baryon resonances that give contribution to photon absorption cross sections. We take into account only five important resonances: $P_{33}(1232), S_{11}(1535), D_{13}(1520), P_{11}(1440), F_{15}(1680)$. Considering the one-pion decay channel of the resonances, the absorption cross sections $\sigma_{1 / 2}$ and $\sigma_{3 / 2}$ may be written as follows [23, 27]:

$$
\sigma_{1 / 2,3 / 2}=\left(\frac{k_{R}}{k}\right)^{2} \frac{W^{2} \Gamma_{\gamma} \Gamma_{R \rightarrow N \pi}}{\left(W^{2}-M_{R}^{2}\right)^{2}+W^{2} \Gamma_{t o t}^{2}} \frac{4 m_{p}}{M_{R} \Gamma_{R}}\left|A_{1 / 2,3 / 2}\right|^{2}
$$

where $A_{1 / 2,3 / 2}$ are transverse electromagnetic helicity amplitudes,

$$
\Gamma_{\gamma}=\Gamma_{R}\left(\frac{k}{k_{R}}\right)^{j_{1}}\left(\frac{k_{R}^{2}+X^{2}}{k^{2}+X^{2}}\right)^{j_{2}}, \quad X=0.3 \mathrm{GeV}
$$

The resonance parameters $\Gamma_{R}, M_{R}, j_{1}, j_{2}, \Gamma_{\text {tot }}$ were taken from [9, 28]. In accordance with Refs. [22, 24, 28] the parameterization of one-pion decay width is

$$
\Gamma_{R \rightarrow N \pi}(q)=\Gamma_{R} \frac{M_{R}}{m_{p}}\left(\frac{q}{q_{R}}\right)^{3}\left(\frac{q_{R}^{2}+C^{2}}{q^{2}+C^{2}}\right)^{2}, \quad C=0.3 \mathrm{GeV}
$$

for the $P_{33}(1232)$ and

$$
\Gamma_{R \rightarrow N \pi}(q)=\Gamma_{R}\left(\frac{q}{q_{R}}\right)^{2 l+1}\left(\frac{q_{R}^{2}+\delta^{2}}{q^{2}+\delta^{2}}\right)^{l+1}
$$

for $D_{13}(1520), P_{11}(1440), F_{15}(1680) . \quad 1$ is the pion angular momentum and $\delta^{2}=$ $\left(M_{R}-m_{p}-m_{\pi}\right)^{2}+\Gamma_{R}^{2} / 4$. Here $\mathrm{k}$ and $\mathrm{q}$ are the photon and pion 3-momentum in the cms for a given center of mass energy W, $k_{R}$ and $q_{R}$ are taken at the pole of the resonance. In the case of $S_{11}(1535)$ we take into account $\pi N$ and $\eta N$ decay modes [24, 28]:

$$
\Gamma_{R \rightarrow \pi, \eta}=\frac{q_{\pi, \eta}}{q} b_{\pi, \eta} \Gamma_{R} \frac{q_{\pi \eta}^{2}+C_{\pi, \eta}^{2}}{q^{2}+C_{\pi, \eta}^{2}},
$$

where $b_{\pi, \eta}$ are the $\pi(\eta)$ branching ratio. 
The cross section $\sigma_{T L}$ is determined by expression similar to (14), containing product $\left(S_{1 / 2}^{*} \cdot A_{1 / 2}+A_{1 / 2}^{*} S_{1 / 2}\right)$ [12]. The calculation of helicity amplitudes $A_{1 / 2}$, $A_{3 / 2}$ and longitudinal amplitude $S_{1 / 2}$, as functions of $Q^{2}$, was done on the basis of constituent quark model in [30, 31, 32, 33, 34]. In the real photon limit $Q^{2}=0$ we take corresponding resonance amplitudes from [9]. For the $\Delta$ - isobar amplitudes $A_{1 / 2}\left(Q^{2}\right), A_{3 / 2}\left(Q^{2}\right)$ we used relations obtained in [35]. Helicity amplitudes of the other resonances were taken from [31, 32, 33, 34]. We have considered Roper resonance $P_{11}(1440)$ as ordinary $q q q$ state. As it follows from predictions of the quark model, the helicity amplitudes, which may be suppressed at $Q^{2}=0$, become dominant very rapidly with $Q^{2}$. It may be seen on Figures 2-5, where we have shown also experimental data of E143 collaboration at two fixed momentum transfer points: $Q^{2} \approx 0.5 \mathrm{GeV}^{2}$ and $Q^{2} \approx 1.2 \mathrm{GeV}^{2}$. Our results for structure function $g_{1}\left(\nu, Q^{2}\right)$ on Figures 2-3, which are in qualitative agreement with [27 and experimental data, show that Breit-Wigner five resonance parameterization of photon cross sections and constituent quark model results give good description of proton polarized structure functions at the resonance region. The existing difference of this model for $g_{1,2}\left(\nu, Q^{2}\right)$ and experimental data, which is particulary seen in Figure 3, demands further improvement in the construction of spin dependent structure functions. This may be done considering contributions of other baryonic resonances in the large $\mathrm{W}$ range: $S_{31}(1620), F_{37}(1950), D_{33}(1700), P_{13}(1720), F_{35}(1905)$ and accounting different decay modes of such states [27]. The sum rule of Gerasimov-Drell-Hern [36]

$$
-\frac{\kappa^{2}}{4 m_{p}^{2}}=\frac{1}{8 \pi^{2} \alpha} \int_{\nu_{t h}}^{\infty} \frac{d \nu}{\nu}\left[\sigma_{1 / 2}(\nu, 0)-\sigma_{3 / 2}(\nu, 0)\right] .
$$

is valid with high accuracy [27]. The second part of (4) gives especially large negative contribution to the correction $\delta_{1}^{P}$ in the range of small $Q^{2}$, where the contribution of $\Delta$ isobar is dominant. With increasing $Q^{2}$ it's value falls and total correction $\delta_{1}^{P}$ has positive sign.

Our calculation of the contribution $\Delta E_{H F S}^{P}$ in the DIS region $\left(W^{2} \geq 4 G e V^{2}\right)$ is based on recent experimental data [12-19]. All of the data, including the SMC data at $Q^{2} \leq 1 G e V^{2}$, were fit to the parameterization:

$$
g_{1}\left(x, Q^{2}\right)=a_{1} x^{a_{2}}\left(1+a_{3} x+a_{4} x^{2}\right)\left[1+a_{5} f\left(Q^{2}\right)\right] F_{1}\left(x, Q^{2}\right),
$$

where $x=Q^{2} / 2 m_{p} \nu$ is the Bjorken scaling variable, $F_{1}=W_{1} m_{p}$. The coefficients of the fits and different models for the form of the $Q^{2}$ dependence may be found in [12, 19]. In Figures 6-7 the experimental data and parameterization in the form (20) for the ratio $g_{1} / F_{1}$ are presented at two different points $Q^{2}$. Numerical integration in (4) was performed with the $f\left(Q^{2}\right)=-\ln Q^{2}$ (fit IV), corresponding to pQCD behavior. We have extrapolated relation (20) to the region near $Q^{2}=0$. Calculation of the second part of correction $\delta^{P}$ in (5) for nonresonance region was performed by means of the Wandzura-Wilczek relation between spin structure functions $g_{1}\left(x, Q^{2}\right)$ and $g_{2}\left(x, Q^{2}\right)$ :

$$
g_{2}\left(x, Q^{2}\right)=-g_{1}\left(x, Q^{2}\right)+\int_{x}^{1} g_{1}\left(t, Q^{2}\right) \frac{d t}{t} .
$$




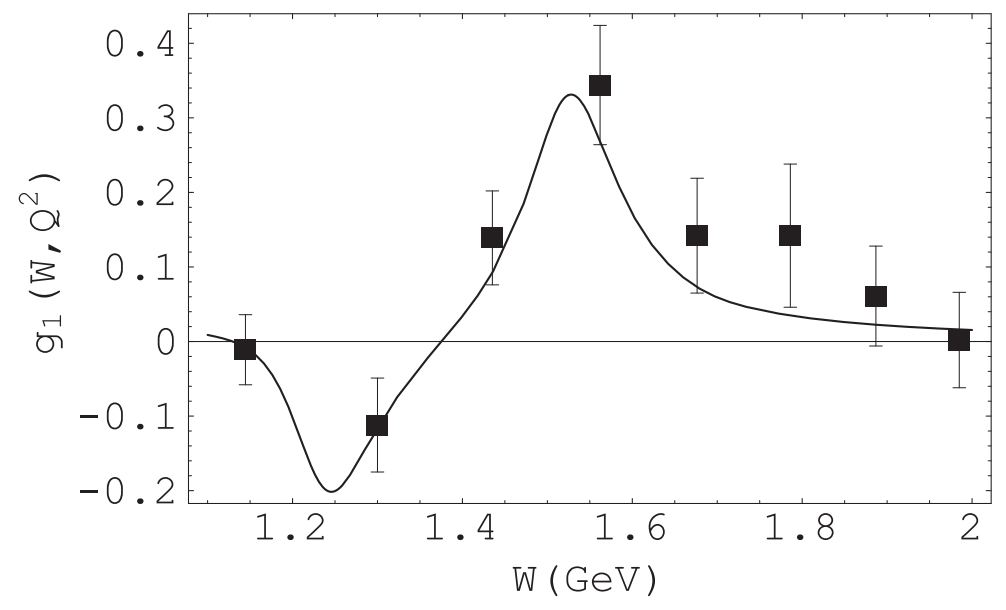

Figure 2: Proton structure function $g_{1}\left(W, Q^{2}\right)$ for $Q^{2}=0.5$ in the resonance region. Experimental points correspond to the paper [12]

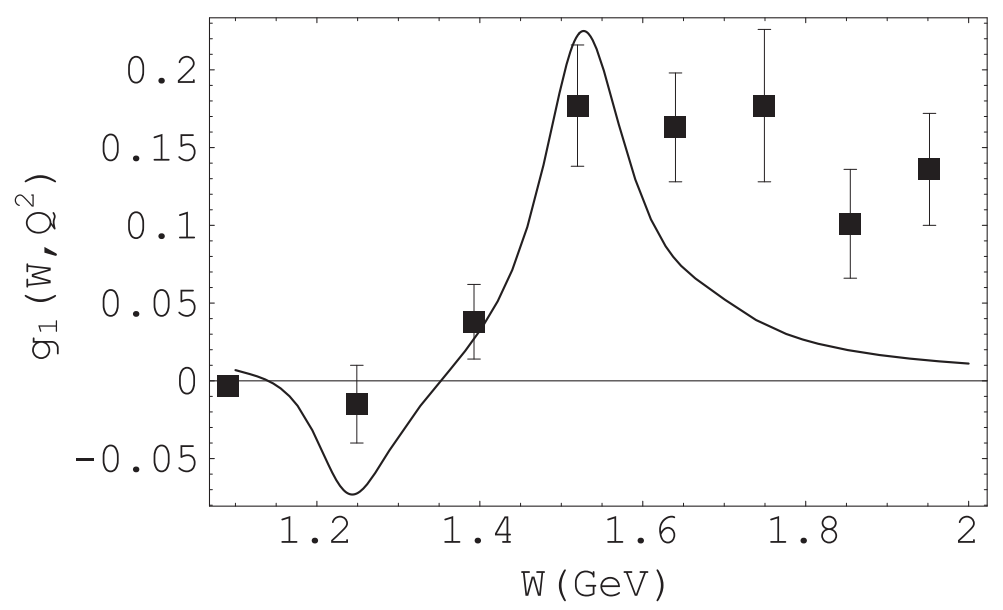

Figure 3: Proton structure function $g_{1}\left(W, Q^{2}\right)$ for $Q^{2}=1.2$ in the resonance region. Experimental points correspond to the paper [12] 


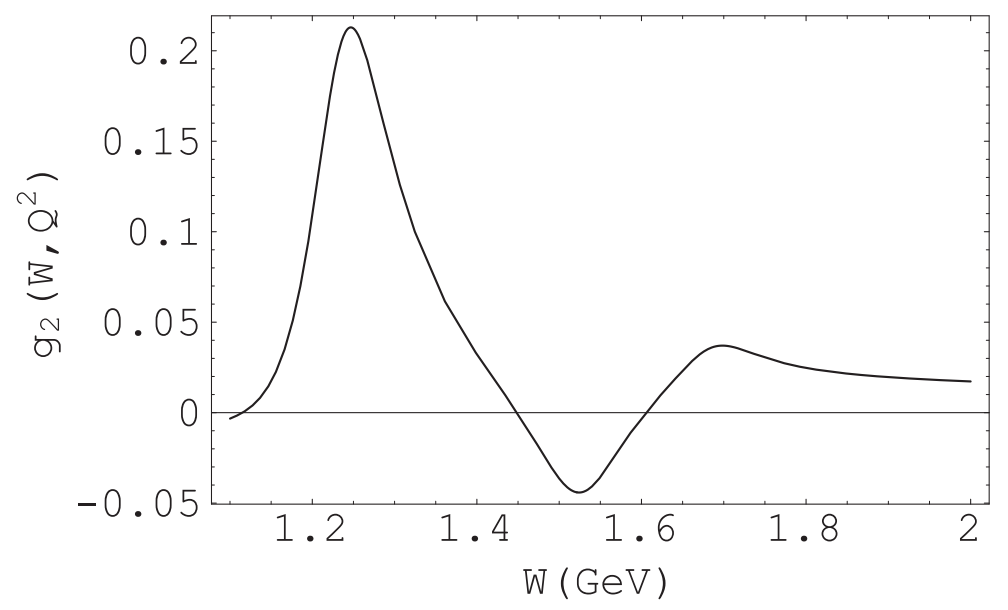

Figure 4: Proton structure function $g_{2}\left(W, Q^{2}\right)$ for $Q^{2}=0.5$ in the resonance region.

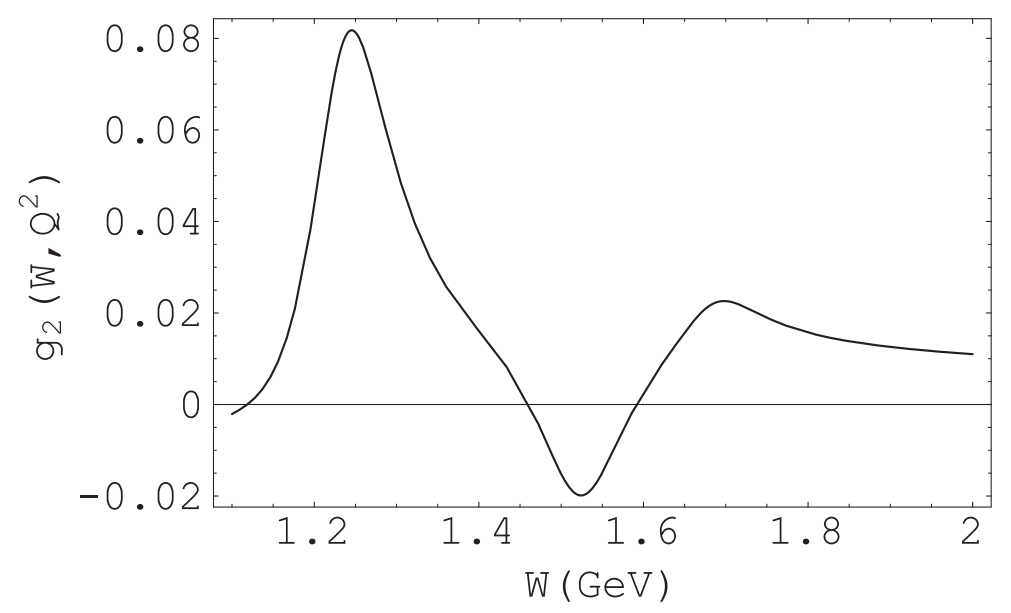

Figure 5: Proton structure function $g_{2}\left(W, Q^{2}\right)$ for $Q^{2}=1.2$ in the resonance region.

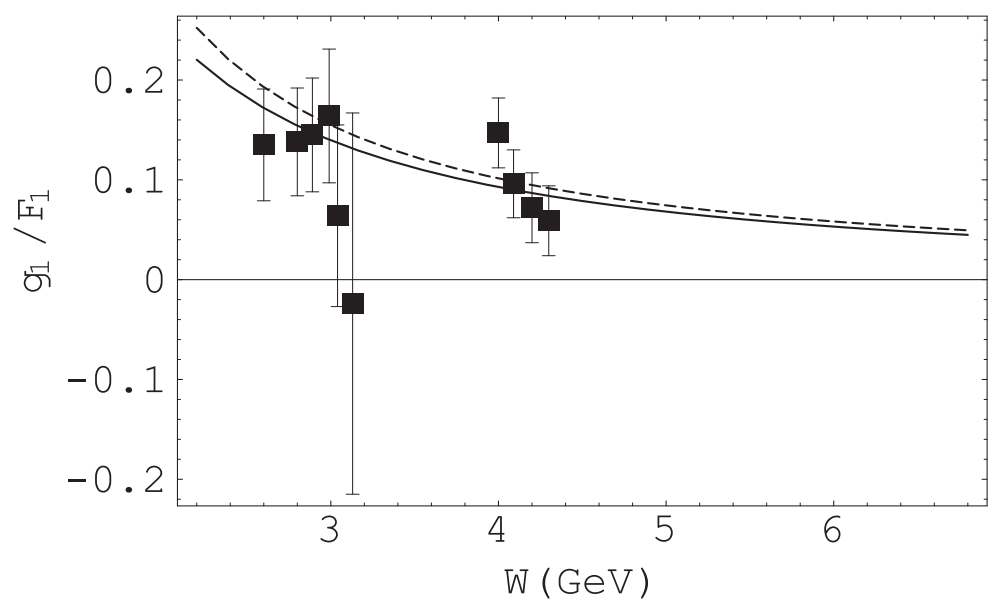

Figure 6: $g_{1}\left(W, Q^{2}\right) / F_{1}\left(W, Q^{2}\right)$ as a function of $\mathrm{W}$ for proton at $Q^{2}=0.7$ in DIS region [12]. The dashed and solid curves are the results of fits I and IV respectively. 


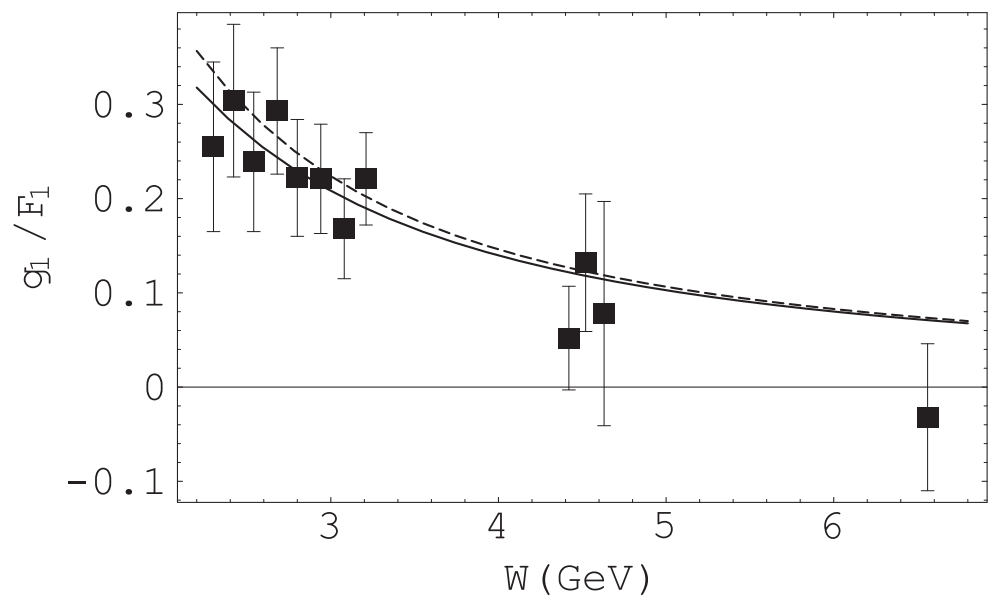

Figure 7: $g_{1}\left(W, Q^{2}\right) / F_{1}\left(W, Q^{2}\right)$ as a function of $\mathrm{W}$ for proton at $Q^{2}=1.2$ in DIS region [12]. The dashed and solid curves are the results of fits I and IV respectively.

The values of contributions $\delta_{1}^{P}, \delta_{2}^{P}$ and total contribution $\delta^{P}$, obtained after the numerical integration in the resonance and nonresonance regions are as follows:

$$
\begin{gathered}
\delta_{1, \text { res }}^{P}=0.93 \mathrm{ppm}, \quad \delta_{1, \text { nonres }}^{P}=0.86 \mathrm{ppm}, \quad \delta_{1}^{P}=1.79 \mathrm{ppm}, \\
\delta_{2, \text { res }}^{P}=-0.42 \mathrm{ppm}, \quad \delta_{2, \text { nonres }}^{P}=-0.01 \mathrm{ppm}, \quad \delta_{2}^{P}=-0.43 \mathrm{ppm}, \\
\delta^{P}=\delta_{1}^{P}+\delta_{2}^{P}=1.4 \pm 0.6 \mathrm{ppm},
\end{gathered}
$$

where the error, indicated in the expression (24), is determined by two main factors, connected with the polarized structure functions: uncertainty of the experimental data in the nonresonance region and possible contribution of the other baryonic resonances to the functions $g_{1,2}\left(\nu, Q^{2}\right)$. Estimation of the second error was done by means of the integration results in (4), (5) for the different intervals of $Q^{2}, \mathrm{~W}$ and possible modification of the spin dependent structure functions in the resonance region $\mathrm{W} \geq 1.5 \mathrm{GeV}$ due to changing of the Breit-Wigner parametrization (14). First part of the error in (24) is connected with statistical and systematical errors of the experimental data from [12].

The difference between the experimental value (1) and the theoretical result $\Delta E_{H F S}^{t h}$ without the proton polarizability contribution can be presented in the form [2, 3, 37, 38]:

$$
\frac{\Delta E_{H F S}^{e x p}-\Delta E_{H F S}^{t h}}{E_{F}}=4.5(1.1) \mathrm{ppm}
$$

As was pointed out in [2, 3, 37, the main sources of uncertainty in this difference are the inaccuracy of the proton form factor parameterization (dipole fit etc.) and the contradictory experimental data on the proton radius. The proton polarizability correction $\delta^{P}$ calculated here gives the contribution (24) of the proper sign and 
order of magnitude to the difference (25). Further improvement of this calculation is connected just as the new experimental and theoretical investigation of the internal structure of the light quark baryons, new more accurate measurements of the proton polarized structure functions, so with the using QCD-based methods of the spin dependent structure functions calculation [40, 41]. More detailed consideration of the structure functions $g_{1,2}\left(\nu, Q^{2}\right)$ at the resonance region, taking into account contributions of some other baryonic resonances and additional decay channels is also needed. This work is in the progress.

We are grateful to M.I. Eides, S.G. Karshenboim, I.B. Khriplovich, V.A. Petrun'kin, R.A. Sen'kov, for useful discussions. The work was performed under the financial support of the Russian Foundation for Fundamental Research (grant 00-02-17771) and the Program "Universities of Russia - Fundamental Researches" (grant 990192).

\section{References}

[1] Hellwig H., Vessot R.F.C., Levine M.W., et al, IEEE Trans. IM-19 (1970) 200.

[2] Dvoeglazov V.V., Tuykhtyaev Yu. N., Faustov R. N. Phys. Part. Nucl. 1994. V.25. No.1. P.58.

[3] Eides M.I., Grotch H., Shelyuto V.A. Theory of light hydrogenlike atoms, hepph/0002158, 2000.

[4] Iddings C.K., Platzman P.M. Phys. Rev. 1959. V.113. P.192.

[5] Drell S.D., Sullivan J.D. Phys. Rev. 1967. V.154. P.1477.

[6] Verganalakis A., Zwanziger D. Nuovo Cimento 1965. V.39. P.613.

[7] Guerin F. Nuovo Cimento 1967. V.A50. P.1.

[8] Zinov'ev G.M., Struminsky B.V., Faustov R.N., et al. Sov. J Nucl. Phys. 1970. V.11. P.715.

[9] Review of Particle Physics EPJ. 1998. V.C3.

[10] Faustov R.N., Martynenko A.P., Saleev V.A. Phys. Atom. Nucl. 1999. V.62. No.12. P.2099.

[11] Close F.E. An introduction to quarks and partons, Academic Press, N.Y., 1979.

[12] Abe K., et al. Preprint SLAC-PUB-7753, 1998.

[13] Abe K., et al. Preprint SLAC-PUB-7242, 1996.

[14] Anthony P.L., et al. Preprint SLAC-PUB-7983, 1999.

[15] Mitchell G.S. Preprint SLAC-PUB-8104, 1999. 
[16] Adams D., et al. Preprint CERN-PPE/97-22, 1997.

[17] Adeva D., et al. Phys. Rev. 1999. V.D60. P.072004.

[18] Hughes V. W. Preprint CERN-PPE/95-178, Aidala C., Deshpande A., Hughes V. In Proc. of the Workshop "Polarized Protons at High Energies - Accelerator challenges and Physics Opportunities", DESY-PROC-1999-03, P.248.

[19] Erbacher R.D. SLAC-Report-546, 1999.

[20] Walker R.L. Phys. Rev. 1969. V.182. P.1729.

[21] Arndt R.A., Workman R.L., Li Z., et al. Phys. Rev. 1990. V.C42. No.5. P.1864.

[22] Teis S., Cassing W., Effenberger M., et al. Z. Phys. 1997. V.A356. P.421.

[23] Effenberger M., Hombach A., Teis S., et al. Nucl. Phys. 1997. V.A613. P.353.

[24] Krusche B., Ahrens J., Anton G., et al. Phys. Rev. Lett. 1995. V.74. No.10. P.3736.

[25] Bianchi N., Muccifora V., Sanctis E., et al. Phys. Rev. 1996. V.C54. No.4. P.1688.

[26] Drechsel D., Hanstein O., Kamalov S.S., et al. Nucl. Phys. 1999. V.A645. P.145.

[27] Dong Y.-B. Eur. Phys. Jour. 1998. V.A1. P.347.

[28] Effenberger M., Hombach A., Teis S., et al. Nucl. Phys. 1997. V.A614. P.501.

[29] Li Z., Dong Y.-B. Phys. Rev. 1996. V.D54. No.7. P.4301.

[30] Koniuk R., Isgur N. Phys. Rev. 1980. V.D21. P.1888.

[31] Close F.E., Li Z. Phys. Rev. 1990. V.D42. No.7. P.2194, P.2207.

[32] Capstick S. Phys. Rev. 1992. V.D46. No.5. P.1965; V.D46. No.7. P.2864.

[33] Li Zhenping, Burkert V., Li Zhujun Phys. Rev. 1992. V.D46. No.1. P.70.

[34] Warns M., Pfeil W., Rollnik H. Phys. Rev. 1990. V.D42. No.7. P.2215.

[35] Carlson C.E., Mukhopadhyay N.C. Preprint RPI-98-N126; WM-98-105; hep$\mathrm{ph} / 9804356$.

[36] Gerasimov S.B. Sov. J. Nucl. Phys. 1966. V.2. P.430.

Drell S.D. Hern A.C. Phys. Rev. Lett. 1966. V.16. P.908.

[37] Bodwin G.T., Yennie D.R. Phys. Rev. 1988. V.D37. P.498. 
[38] Karshenboim S.G. Phys. Lett. 1997. V.A225. P.97.

[39] Burkert V. Nucl. Phys. 1997. V.A623. P.59.

[40] Altarelli G., Ball R.D., Forte S., et al. Nucl. Phys. 1997. V.B496. P.337.

[41] Hirai M., Kumano S., Miyama M. Comp. Phys. Comm. 1998. V.108. P.38. 